\title{
HiBISCuS: Hypergraph-Based Source Selection for SPARQL Endpoint Federation
}

\author{
Muhammad Saleem and Axel-Cyrille Ngonga Ngomo \\ Universität Leipzig, IFI/AKSW, PO 100920, D-04009 Leipzig \\ \{lastname informatik.uni-leipzig.de
}

\begin{abstract}
Efficient federated query processing is of significant importance to tame the large amount of data available on the Web of Data. Previous works have focused on generating optimized query execution plans for fast result retrieval. However, devising source selection approaches beyond triple pattern-wise source selection has not received much attention. This work presents HiBISCuS, a novel hypergraph-based source selection approach to federated SPARQL querying. Our approach can be directly combined with existing SPARQL query federation engines to achieve the same recall while querying fewer data sources. We extend three well-known SPARQL query federation engines with HiBISCus and compare our extensions with the original approaches on FedBench. Our evaluation shows that HiBISCuS can efficiently reduce the total number of sources selected without losing recall. Moreover, our approach significantly reduces the execution time of the selected engines on most of the benchmark queries.
\end{abstract}

\section{Introduction}

The Web of Data is now a large compendium of interlinked data sets from multiple domains with large datasets [12] being added frequently [3]. Given the complexity of information needs on the Web, certain queries can only be answered by retrieving results contained across different data sources (short: sources). Thus, the optimization of engines that support this type of queries, called federated query engines, is of central importance to ensure the usability of the Web of Data in real-world applications. One of the important optimization steps in federated SPARQL query processing is the efficient selection of relevant sources for a query. To ensure that a recall of $100 \%$ is achieved, most SPARQL query federation approaches $[4,8,10,14,15,11]$ perform triple pattern-wise source selection (TPWSS). The goal of the TPWSS is to identify the set of relevant (also called capable, formally defined in section 3 ) sources against individual triple patterns of a query [11]. However, it is possible that a relevant source does not contribute to the final result set of the complete query. This is because the results from a particular data source can be excluded after performing joins with the results of other triple patterns contained in the same query. An overestimation of such sources increases the network traffic and can significantly affect the overall query processing time.

An example for such a query is SSQ1 in Figure 1. A TPWSS that retrieves all relevant sources for each individual triple pattern would lead to all sources in the example being queried. Yet, the complete result set of SSQ1 can be computed without querying $d_{2}$ due to the type of join used in the query. We thus propose a novel join-aware approach to 


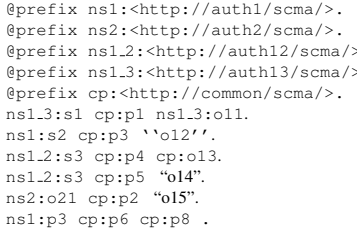

(a) Dataset $d_{1}$

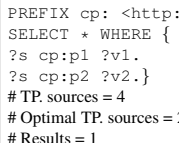

(d) Star-shaped query SSQ1

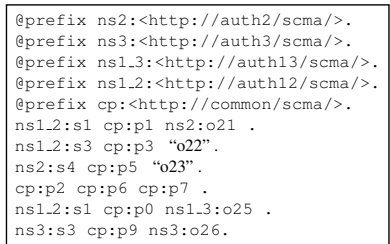

(b) Dataset $d_{2}$

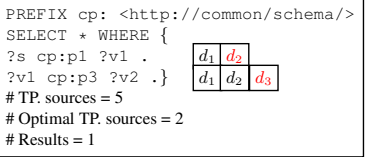

(e) Path-shaped query PSQ2

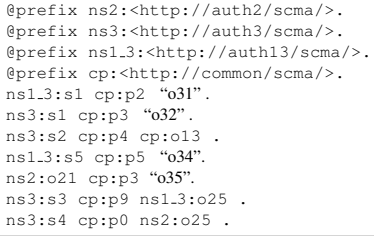

(c) Dataset $d_{3}$

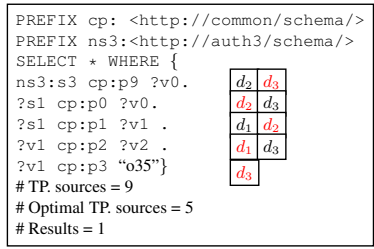

(f) Hybrid query HSQ3

Fig. 1: Motivating Examples. \#TP is the total number of triple pattern-wise sources selected. The relevant sources for each are shown next to each triple pattern. The sources marked in red contribute to the final query result set.

TPWSS dubbed HiBISCuS. Our approach goes beyond the state of the art by aiming to compute the sources that actually contribute to the final result set of an input query and that for each triple pattern. To the best of our knowledge, this join-aware approach to TPWSS has only been tackled by an extension of the ANAPSID framework presented in [9]. Yet, this extension is based on evaluating namespaces and sending ASK queries to data sources at runtime. In contrast, HiBISCuS relies on an index that stores the authorities of the resource URIs ${ }^{1}$ contained in the data sources at hand. Our approach proves to be more time-efficient than the ANAPSID extension as shown by our evaluation in Section 5.

HiBISCuS addresses the problem of source selection by several innovations. Our first innovation consists of modelling SPARQL queries as a sets of directed labelled hypergraphs (DLH). Moreover, we rely on a novel type of summaries which exploits the fact that the resources in SPARQL endpoints are Uniform Resource Identifiers (URIs). Our source selection algorithm is also novel and consists of two steps. In a first step, our approach labels the hyperedges of the DLH representation of an input SPARQL query $q$ with relevant data sources. In the second step, the summaries and the type of joins in $q$ are used to prune the edge labels. By these means, HiBISCuS can discard sources (without losing recall) that are not pertinent to the computation of the final result set of the query. Overall, our contributions are thus as follows:

1. We present a formal framework for modelling SPARQL queries as directed labelled hypergraphs.

2. We present a novel type of data summaries for SPARQL endpoints which relies on the authority fragment of URIs.

3. We devise a pruning algorithm for edge labels that enables us to discard irrelevant sources based on the types of joins used in a query.

\footnotetext{
${ }^{1}$ http://tools.ietf.org/html/rfc3986
} 
4. We evaluate our approach by extending three state-of-the-art federate query engines (FedX, SPLENDID and DARQ) with HiBISCuS and comparing these extensions to the original systems. In addition, we compare our most time-efficient extension with the extension of ANAPSID presented in [9]. Our results show that we can reduce the number of source selected, the source selection time as well as the overall query runtime of each of these systems.

The structure of the rest of this paper is as follows: we first give a brief overview of federated query engines. Then, we present our formalization of SPARQL queries as directed labelled hypergraphs. The algorithms underlying HiBISCuS are then explained in detail. Finally, we evaluate HiBISCuS against the state-of-the-art and show that we achieve both better source selection and runtimes on the FedBench [13] SPARQL query federation benchmark.

\section{Related Work}

The approaches related to query federation over the Web of Data can be divided into three main categories (see Table 1 obtained from our public survey results ${ }^{2}$ ).

(1) Query federation approaches over multiple SPARQL endpoints make use of the SPARQL endpoints due to which they provide a time-efficient solution to SPARQL query federation. However, the RDF data needs to be exposed as SPARQL endpoints. Due to which they are unable to deal with whole LOD. (2) Query federation over Linked Data do not require the data to be exposed via SPARQL endpoints. The only requirement is that it should follow the Linked Data principles. ${ }^{3}$ Due to URI lookups at runtime, these type of approaches are commonly slower than the previous type of approaches. (3) Query federation approaches on top of Distributed Hash Tables store the RDF data on top of Distributed Hash Tables (DHTs). This is a space-efficient solution and can reduce the network cost as well. However, an important fraction of the LOD datasets is not stored using DHTs.

The source selection approaches used in each of the categories can further divided into three sub-categories(see Table 1). (1)Catalog/index-assisted source selection only makes use of an index/data catalog (also called data summaries) to perform TPWSS. The result completeness (100\% recall) must be ensured by keeping the index up-to-date. (2)Catalog/index-free source selection approaches do not make use of any pre-stored index and can thus always compute complete and up-to-date records. However, they commonly have a longer query execution time due to the extra processing required for collecting on-the-fly statistics (e.g. SPARQL ASK operations). (3) Hybrid source selection approaches are a combination of the previous approaches.

In this paper, we propose a novel hybrid source selection approach for SPARQL endpoint federation systems dubbed HiBISCuS. In contrast to the state of the art, HiBISCuS uses hypergraphs to detect sources that will not generate any relevant results both at triple-pattern level and at query level. By these means, HiBISCuS can generate better approximations of the sources that should be queried to return complete results for a given query.

\footnotetext{
${ }^{2}$ Survey: http://goo.gl/iXvKVT, Results: http://goo.gl/CNW5UC

${ }^{3}$ http://www.w3.org/DesignIssues/LinkedData.html
} 
Table 1: Classification of SPARQL federation engines. ( $\mathbf{S E F}=$ SPARQL Endpoints Federation, $\mathbf{D H T}=$ DHT Federation, $\mathbf{L D F}=$ Linked Data Federation, $\mathbf{C t g} .=$ Federation Type, $\mathbf{F d X}=$ FedX, SPL =SPLENDID, ADE = ADERIS, I.F = Index-free, I.O = Indexonly, HB = Hybrid, C.A. = Code Availability, S.S.T. $=$ Source Selection Type, I.U. $=$ Index Update, $\mathbf{N A}=$ Not Applicable, $(\mathbf{A}+\mathbf{I})=$ SPARQL ASK and Index,$(\mathbf{C}+\mathbf{L})=$ Catalog and online discovery via Link-traversal, *only source selection approaches.)

\begin{tabular}{|c|c|c|c|c|c|c|c|c|c|c|c|c|}
\hline & $\begin{array}{c}\text { FedX } \\
{[14]}\end{array}$ & $\begin{array}{c}\text { LHD } \\
{[15]}\end{array}$ & $\begin{array}{l}\text { SPL } \\
{[4]}\end{array}$ & $\begin{array}{c}\text { DAW* } \\
\text { [11] }\end{array}$ & $\begin{array}{c}\text { ANAPSID } \\
{[1]}\end{array}$ & $\begin{array}{c}\mathrm{ADE} \\
{[8]}\end{array}$ & \begin{tabular}{|c|} 
DARQ \\
{$[10]$}
\end{tabular} & $\begin{array}{c}\text { LDQP } \\
\text { [7] }\end{array}$ & $\begin{array}{c}\text { WoDQA } \\
{[2]}\end{array}$ & $\begin{array}{c}\text { Atlas } \\
{[6]}\end{array}$ & $\begin{array}{c}\text { QTree* } \\
{[5]}\end{array}$ & HiBISCus* \\
\hline Ctg. & SEF & SEF & SEF & - & SEF & SEF & SEF & LDF & LDF & DHT & - & - \\
\hline C.A & $\checkmark$ & $\checkmark$ & $\checkmark$ & $x$ & $\checkmark$ & $\checkmark$ & $\checkmark$ & $x$ & $\checkmark$ & $\checkmark$ & $x$ & $\checkmark$ \\
\hline S.S.T & I.F & $\mathrm{HB}(\mathrm{A}+\mathrm{I})$ & $\mathrm{HB}(\mathrm{A}+\mathrm{I})$ & $\mathrm{HB}(\mathrm{A}+\mathrm{I})$ & $\mathrm{HB}(\mathrm{A}+\mathrm{I})$ & I.O & I.O & $\mathrm{HB}(\mathrm{C}+\mathrm{L})$ & $\mathrm{HB}(\mathrm{A}+\mathrm{I})$ & I.O & I.O & $\mathrm{HB}(\mathrm{A}+\mathrm{I})$ \\
\hline Cache & $\checkmark$ & $x$ & $x$ & $\checkmark$ & $x$ & $x$ & $x$ & $x$ & $\checkmark$ & $x$ & $x$ & $\checkmark$ \\
\hline I.U & NA & $x$ & $x$ & $x$ & $\checkmark$ & $x$ & $x$ & $x$ & $\checkmark$ & $x$ & $\checkmark$ & $\checkmark$ \\
\hline
\end{tabular}

\section{Preliminaries}

In the following, we present some of the concepts and notation that are used throughout this paper. RDF resources are identified by using a Unified Resource Identifier (URI). Each URI has a generic syntax consists of a hierarchical sequence of components namely the scheme, authority, path, query, and fragment ${ }^{4}$. For example, the prefix $n s 1=$ $<h t t p: / /$ auth $1 /$ scma/> used in Figure 1 consist of scheme $h t t p$, authority auth 1 , and path scma. The details of the remaining two components are out of the scope of this paper. In the rest of the paper, we jointly refer to the first two components (path, authority) as authority of a URI.

The standard for querying RDF is SPARQL. ${ }^{5}$ The result of a SPARQL query is called its result set. Each element of the result set of a query is a set of variable bindings. Federated SPARQL queries are defined as queries that are carried out over a set of sources $\mathrm{D}=\left\{d_{1}, \ldots, d_{n}\right\}$. Given a SPARQL query $q$, a source $d \in D$ is said to contribute to $q$ if at least one of the variable bindings belonging to an element of $q$ 's result set can be found in $d$.

Definition 1 (Relevant source Set). A source $d \in D$ is relevant (also called capable) for a triple pattern $t p_{i} \in T P$ if at least one triple contained in $d$ matches $t p_{i} \cdot{ }^{6}$ The relevant source set $R_{i} \subseteq D$ for tp $p_{i}$ is the set that contains all sources that are relevant for that particular triple pattern.

For example, the set of relevant sources for the triple pattern $<? s, \mathrm{cp}: \mathrm{p} 1, ? v 1>$ of SSQ1 is $\left\{d_{1}, d_{2}\right\}$. It is possible that a relevant source for a triple pattern does not contribute to the final result set of the complete query $q$. This is because the results computed from a particular source $d$ for a triple pattern $t p_{i}$ might excluded while performing joins with the results of other triple patterns contained in the query $q$. For example, consider SSQ1. The results from $d_{2}$ for $<? s, \mathrm{cp}: \mathrm{p} 1, ? v 1>$ and $d_{3}$ for

\footnotetext{
${ }^{4}$ URI syntax: http://tools.ietf.org/html/rfc3986

${ }^{5} \mathrm{http}: / /$ www.w3.org/TR/rdf-sparql-query/

${ }^{6}$ The concept of matching a triple pattern is defined formally in the SPARQL specification found at http://www.w3.org/TR/rdf-sparql-query/
} 
$<? s, \mathrm{cp}: \mathrm{p} 2, ? v 1>$ are excluded after performing the join between the results of the two triple patterns.

Definition 2 (Optimal source Set). The optimal source set $O_{i} \subseteq R_{i}$ for a triple pattern $t p_{i} \in T P$ contains the relevant sources $d \in R_{i}$ that actually contribute to computing the complete result set of the query.

For example, the set of optimal sources for the triple pattern $<? s, \mathrm{cp}: \mathrm{p} 2, ? v 2>$ of SSQ1 is $\left\{d_{3}\right\}$, while the set of relevant sources for the same triple pattern is $\left\{d_{1}, d_{3}\right\}$. Formally, the problem of TPWSS can then be defined as follows:

Definition 3 (Problem Statement). Given a set D of sources and a query q, find the optimal set of sources $O_{i} \subseteq D$ for each triple pattern t $p_{i}$ of $q$.

Most of the source selection approaches $[4,8,10,14,15]$ used in SPARQL endpoint federation systems only perform TPWSS, i.e., they find the set of relevant sources $R_{i}$ for individual triple patterns of a query and do not consider computing the optimal source sets $O_{i}$. In this paper, we present an index-assisted approach for (1) the time-efficient computation of relevant source set $R_{i}$ for individual triple patterns of the query and (2) the approximation of $O_{i}$ out of $R_{i}$. HiBISCuS approximates $O_{i}$ by determining and removing irrelevant sources from each of the $R_{i}$. We denote our approximation of $O_{i}$ by $R S_{i}$. HiBISCuS relies on directed labelled hypergraphs (DLH) to achieve this goal. In the following, we present our formalization of SPARQL queries as DLH. Subsequently, we show how we make use of this formalization to approximate $O_{i}$ for each $t p_{i}$.

\section{HiBISCuS}

In this section we present our approach to the source selection problem in details. We begin by presenting our approach to representing BGPs ${ }^{7}$ of a SPARQL query as DLHs. Then, we present our approach to computing lightweight data summaries. Finally, we explain our approach to source selection.

\subsection{Queries as Directed Labelled Hypergraphs}

An important intuition behind our approach is that each of the BGP in a query can be executed separately. Thus, in the following, we will mainly focus on how the execution of a single BGP can be optimized. The representation of a query as DLH is the union of the representations of its BGPs. Note that the representations of BGPs are kept disjoint even if they contain the same nodes to ensure that the BGPs are processed independently. The DLH representation of a BGP is formally defined as follows:

Definition 4. Each basic graph patterns $B G P_{i}$ of a SPARQL query can be represented as a $D L H H G_{i}=\left(V, E, \lambda_{e}, \lambda_{v t}\right)$, where

1. $V=V_{s} \cup V_{p} \cup V_{o}$ is the set of vertices of $H G_{i}$, $V_{s}$ is the set of all subjects in $H G_{i}$, $V_{p}$ the set of all predicates in $H G_{i}$ and $V_{o}$ the set of all objects in $H G_{i}$;

\footnotetext{
${ }^{7}$ http://www.w3.org/TR/sparq111-query/\#BasicGraphPatterns
} 


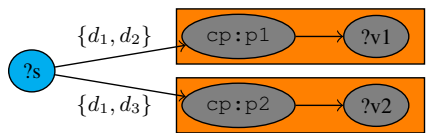

$V_{s}=\{? s\}, V_{p}=\{\mathrm{cp}: \mathrm{p} 1, \mathrm{cp}: \mathrm{p} 2\}, V_{o}=\{? v 1, ? v 2\}$

$V=V_{s} \cup V_{p} \cup V_{o}=\{? s, \mathrm{cp}: \mathrm{p} 1, \mathrm{cp}: \mathrm{p} 2, ? v 1, ? v 2\}$,

$E=\left\{e_{1}, e_{2}\right\}, e_{1}=(? s, \mathrm{cp}: \mathrm{p} 1, ? v 1), e 2=(? s, \mathrm{cp}: \mathrm{p} 2, ? v 2)$,

$\lambda_{e}\left(e_{1}\right)=\left\{d_{1}, d_{2}\right\}, \lambda_{e}\left(e_{2}\right)=\left\{d_{1}, d_{3}\right\}$

(a) SSQ1
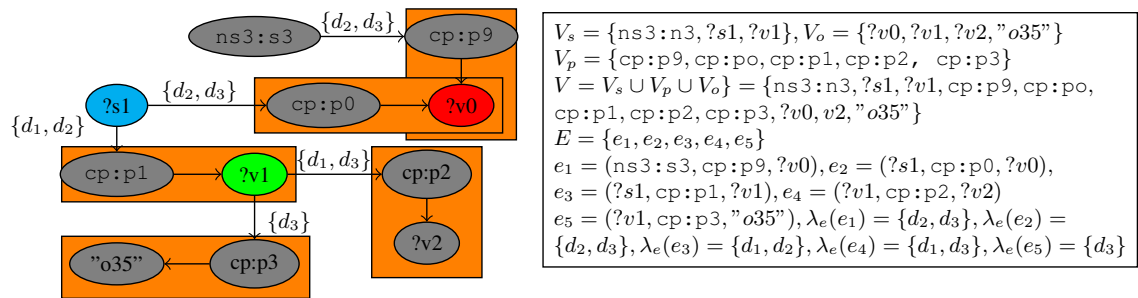

(b) HSQ3

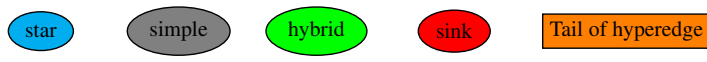

Fig. 2: Labelled hypergraph of query SSQ1 and query HSQ3 of Figure 1

2. $E=\left\{e_{1}, \ldots, e_{t}\right\} \subseteq V^{3}$ is a set of directed hyperedges (short: edge). Each edge $e=$ $\left(v_{s}, v_{p}, v_{o}\right)$ emanates from the triple pattern $\left\langle v_{s}, v_{p}, v_{o}>\right.$ in $B G P_{i}$. We represent these edges by connecting the head vertex $v_{s}$ with the tail hypervertex $\left(v_{p}, v_{o}\right)$. In addition, we use $E_{i n}(v) \subseteq E$ and $E_{\text {out }}(v) \subseteq E$ to denote the set of incoming and outgoing edges of a vertex $v$;

3. $\lambda_{e}: E \mapsto 2^{D}$ is a hyperedge-labelling function. Given a hyperedge $e \in E$, its edge label is a set of sources $R_{i} \subseteq D$. We use this label to the sources that should be queried to retrieve the answer set for the triple pattern represented by the hyperedge $e$;

4. $\lambda_{v t}$ is a vertex-type-assignment function. Given an vertex $v \in V$, its vertex type can be 'star', 'path', 'hybrid', or 'sink' if this vertex participates in at least one join. A 'star' vertex has more than one outgoing edge and no incoming edge. 'path' vertex has exactly one incoming and one outgoing edge. A 'hybrid' vertex has either more than one incoming and at least one outgoing edge or more than one outgoing and at least one incoming edge. A 'sink' vertex has more than one incoming edge and no outgoing edge. A vertex that does not participate in any join is of type 'simple'.

Figure 2a shows the hypergraph of SSQ1 and Figure 2b represents the hypergraph of HSQ3 of motivating example given in Figure 1. We can now reformulate our problem statement as follows:

Definition 5 (Problem Reformulation). Given a query $q$ represented as a set of hypergraphs $\left\{H G_{1}, \ldots, H G_{x}\right\}$, find the labelling of the hyperedges of each hypergraph $H G_{i}$ that leads to an optimal source selection.

\subsection{Data Summaries}

HiBISCuS relies on capabilities to compute data summaries. Given a source $d$, we define a capability as a triple $(p, S A(d, p), O A(d, p))$ which contains (1) a predicate $p$ in $d,(2)$ 
Listing 1.1: HiBISCuS example. Prefixes are ignored for simplicity

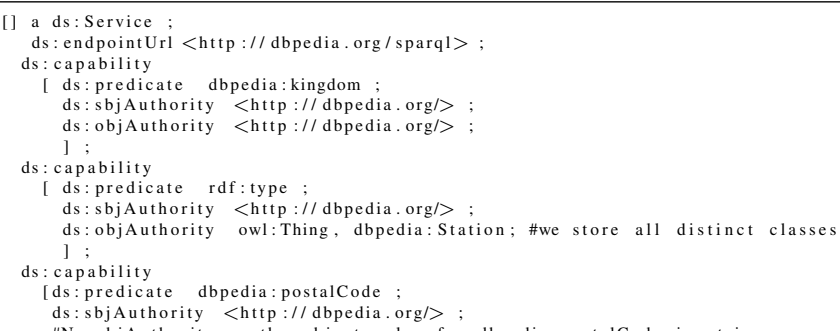

the set $S A(d, p)$ of all distinct subject authorities (ref. section 3) of $p$ in $d$ and (3) the set $O A(d, p)$ of all distinct object authorities of $p$ in $d$. In HiBISCuS, a data summary for a source $d \in D$ is the set $C A(d)$ of all capabilities of that source. Consequently, the total number of capabilities of a source is equal to the number of distinct predicates in it.

The predicate $r d f: t y p e$ is given a special treatment: Instead of storing the set of all distinct object authorities for a capability having this predicate, we store the set of all distinct class URIs in $d$, i.e., the set of all resources that match $? x$ in the query ? y rdf: type ?x. The reason behind this choice is that the set of distinct classes used in a source $d$ is usually a small fraction of the set of all resources in $d$. Moreover, triple patterns with predicate $r d f: t y p e$ are commonly used in SPARQL queries. Thus, by storing the complete class URI instead of the object authorities, we might perform more accurate source selection. Listing 1.1 shows an example of a data summary. In the next section, we will make use of these data summaries to optimize the TPWSS.

\subsection{Source Selection Algorithm}

Our source selection comprise two steps: given a query $q$, we first label all hyperedges in each of the hypergraphs which results from the BGPs of $q$, i.e., we compute $\lambda_{e}\left(e_{i}\right)$ for each $e_{i} \in E_{i}$ in all $H G_{i} \in D H G$. We present two variations of this step and compare them in the evaluation section. In a second step, we prune the labels of the hyperedges assigned in the first step and compute $R S_{i} \subseteq R_{i}$ for each $e_{i}$. The pseudo-code of our approaches is shown in Algorithms 1, 2 (labelling) as well as 3 (pruning).

Labelling approaches We devised two versions of our approach to the hyperedge labelling problem, i.e., an ASK-dominant and an index-dominant version. Both take the set of all sources $D$, the set of all disjunctive hypergraphs $D H G$ of the input query $q$ and the data summaries $H i B I S C u S_{D}$ of all sources in $D$ as input (see Algorithms 1,2). They return a set of labelled disjunctive hypergraphs as output. For each hypergraph and each hyperedge, the subject, predicate, object, subject authority, and object authority are collected (Lines 2-5 of Algorithms 1,2). Edges with unbound subject, predicate, and object vertices (e.g e $=(? \mathrm{~s}, ? \mathrm{p}, ? \mathrm{o}))$ are labelled with the set of all possible sources $D$ (Lines 6-7 of Algorithms 1,2). A data summary lookup is performed for edges with the 


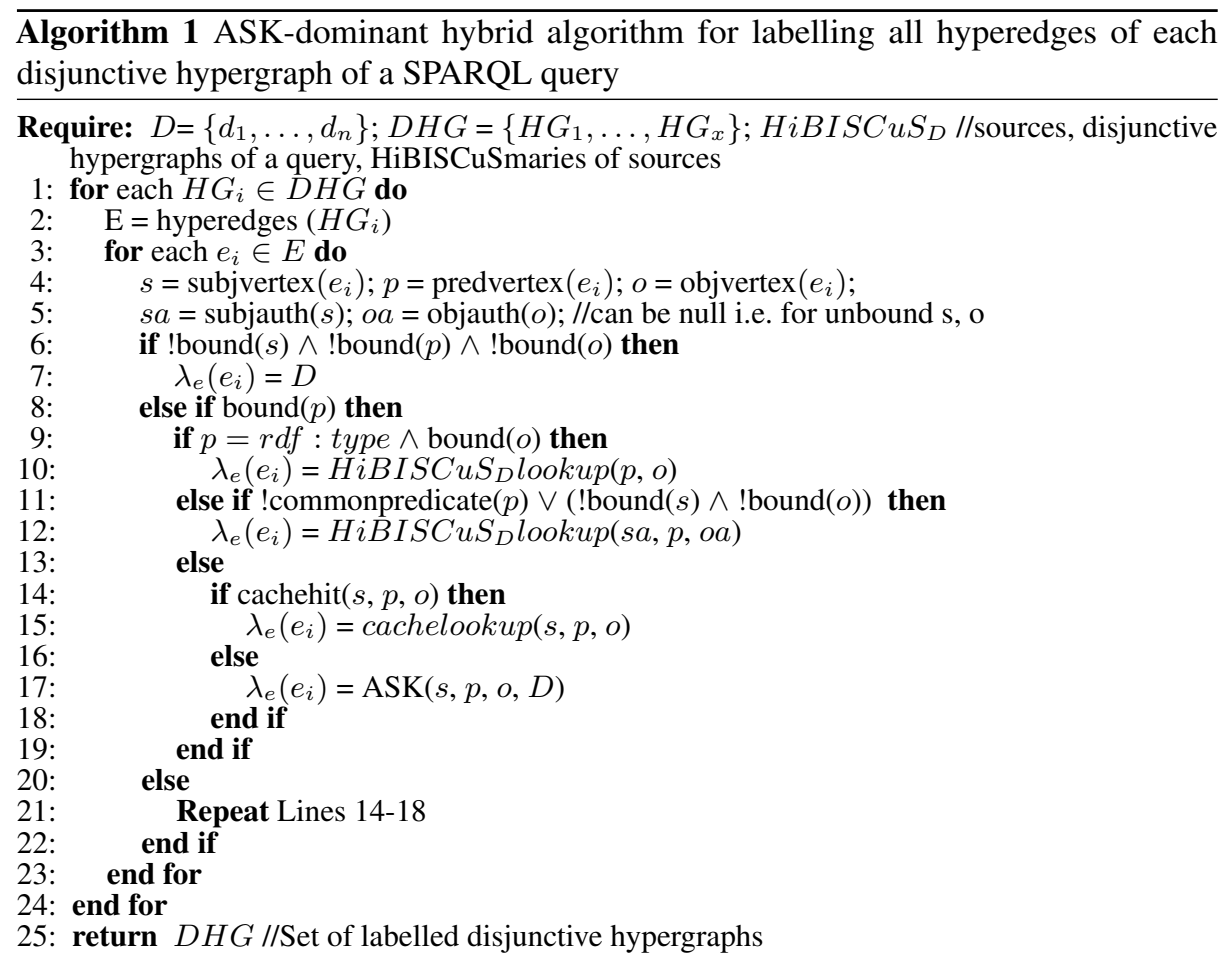

predicate vertex $r d f:$ type that have a bound object vertex. All sources with matching capabilities are selected as label of the hyperedge (Lines 9-10 of Algorithms 1,2).

The ASK-dominant version of our approach (see Algorithm 1, Line 11) makes use of the notion of common predicates. A common predicate is a predicate that is used in a number of sources above a specific threshold value $\theta$ specified by the user. A predicate is then considered a common predicate if it occurs in at least $\theta|D|$ sources. We make use of the ASK queries for triple patterns with common predicates. Here, an ASK query is sent to all of the available sources to check whether they contain the common predicate $c p$. Those sources which return true are selected as elements of the set of sources used to label that triple pattern. The results of the ASK operations are stored in a cache. Therefore, every time we perform a cache lookup before SPARQL ASK operations (Lines 14-18). In contrast, in the index-dominant version of our algorithm, an index lookup is performed if any of the subject or predicate is bound in a triple pattern.We will see later that the index-dominant approach requires less ASK queries than the ASK-dominant algorithm. However, this can lead to an overestimation of the set of relevant sources (see section 5.2).

\subsection{Pruning approach}

The intuition behind our pruning approach is that knowing which authorities are relevant to answer a query can be used to discard triple pattern-wise (TPW) selected sources that will not contribute to the final result set of the query. Our source pruning algorithm (ref. 


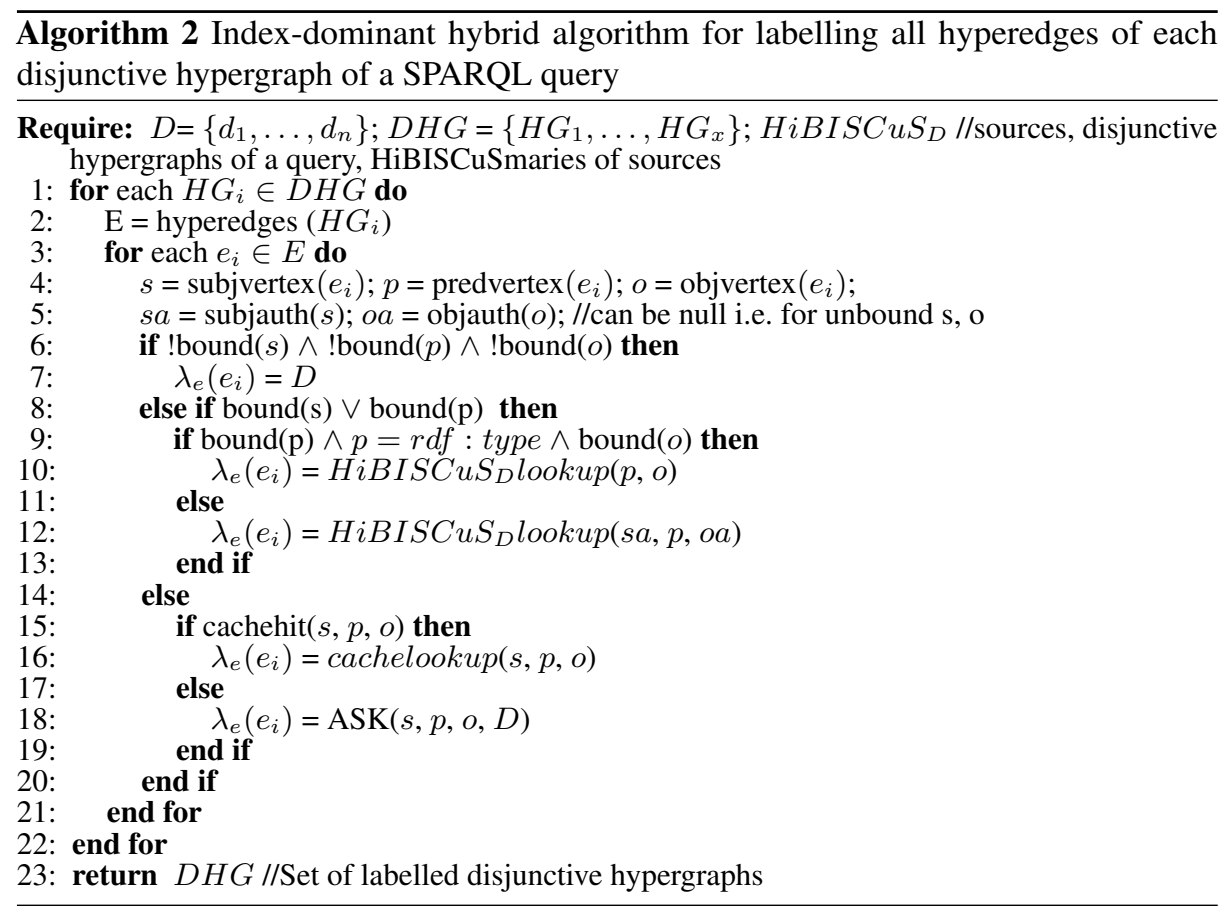

Algorithm 3) takes the set of all labelled disjunctive hypergraphs as input and prune labels of all hyperedges which either incoming or outgoing edges of a 'star','hybrid', 'path', or 'sink' node. Note that our approach deals with each BGP of the query separately (Line 1 of Algorithm 3).

For each node $v$ of a DLH that is not of type 'simple', we first retrieve the sets (1) SAuth of the subject authorities contained in the elements of the label of each outgoing edge of $v$ (Lines 5-7 of Algorithm 3) and (2) OAuth of the object authorities contained in the elements of the label of each ingoing edge of $v$ (Lines 8-10 of Algorithm 3). Note that these are sets of sets of authorities. For the node ? $v 1$ of HSQ3 in our running example (see Figure 3), we get $S A$ uth $=\{$ auth13, auth2 $\}$ for the ingoing edge and $O A u t h=\{\{$ auth13, auth2 $\},\{$ auth2 $\}\}$ for the outgoing edges. Now we merge these two sets to the set $A$ of all authorities. For node ?v1 in HSQ3, $A=\{\{$ auth13, auth2 $\},\{$ auth13, auth2 $\},\{$ auth2 $\}$. The intersection $I=\left(\bigcap_{a_{i} \in A} a_{i}\right)$ of these elements sets is then computed. In our example, this results in $I=\{$ auth2 $\}$. Finally, we recompute the label of each hyperedge $e$ that is connected to $v$. To this end, we compute the subset of the previous label of $e$ which is such that the set of authorities of each of its elements is not disjoint with $I$ (see Lines 16-23 of Algorithm 3). These are the only sources that will really contribute to the final result set of the query.

We are sure not to lose any recall by this operation because joins act in a conjunctive manner. Consequently, if the results of a data source $d_{i}$ used to label a hyperedge cannot be joined to the results of at least one source of each of the other hyperedges, it is 


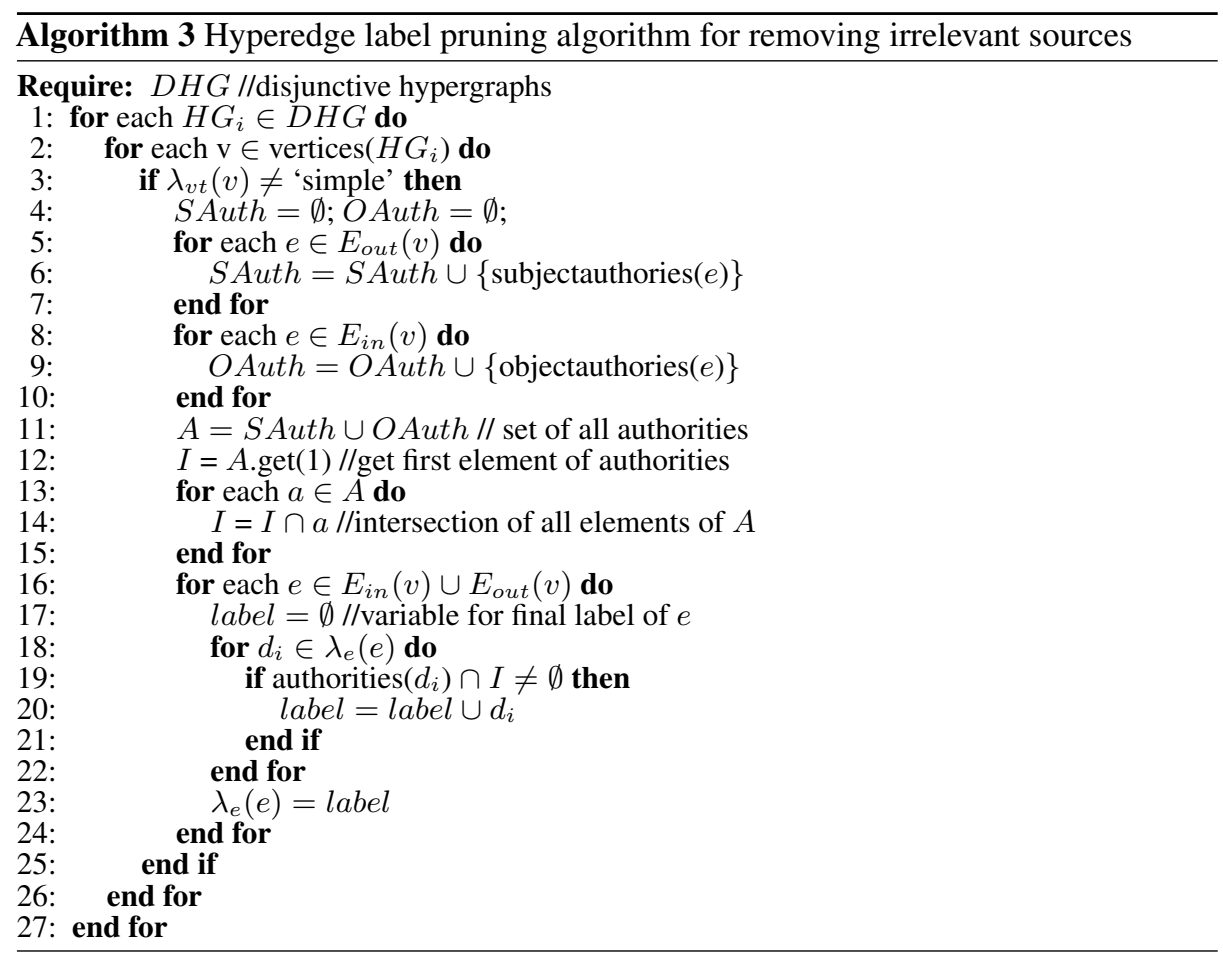

guaranteed that $d_{i}$ will not contribute to the final result set of the query. In our example, this leads to $d_{1}$ being discarded from the label of the ingoing edge, while $d_{3}$ is discarded from the label of one outgoing hyperedge of node ? $v 1$ as shown in Figure 3 . This step concludes our source selection.

\section{Evaluation}

In this section we describe the experimental evaluation of our approach. We first describe our experimental setup in detail. Then, we present our evaluation results. All data used in this evaluation is either publicly available or can be found at the project web page. ${ }^{8}$

\subsection{Experimental Setup}

Benchmarking Environment: We used FedBench [13] for our evaluation. It is the only (to the best of our knowledge) benchmark that encompasses real-world datasets and commonly used queries within a distributed data environment. Furthermore, it is commonly used in the evaluation of SPARQL query federation systems $[14,4,9,11]$. Each of FedBench's nine datasets was loaded into a separate physical virtuoso server. The exact specifications of the servers can be found on the project website. All experiments were ran on a machine with a $2.70 \mathrm{GHz}$ i5 processor, $8 \mathrm{~GB}$ RAM and $300 \mathrm{~GB}$ hard

\footnotetext{
${ }^{8}$ https://code.google.com/p/hibiscusfederation/
} 


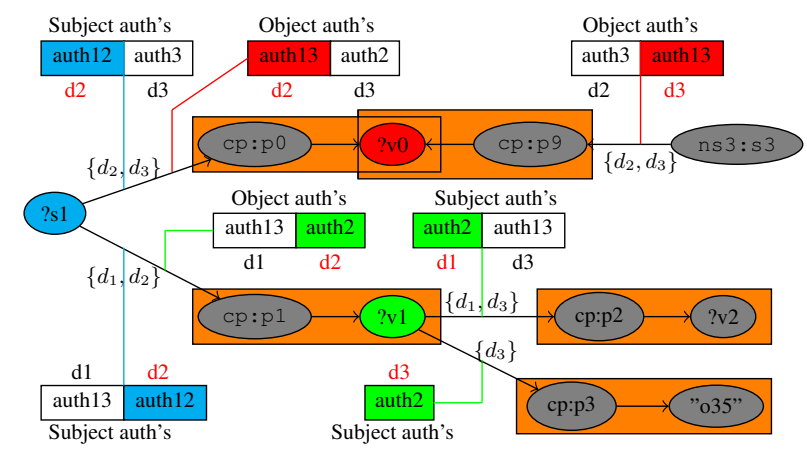

Fig. 3: Source pruning of Labeled hypergraph HSQ3 of Figure 1. All the sources highlighted in red are finally selected

disk. The experiments were carried out in a local network, so the network costs were negligible. Each query was executed 10 times and results were averaged. The query timeout was set to 30min (1800s). The threshold for the ASK-dominant approach was best selected to 0.33 after analysing results of different threshold values.

Federated Query Engines: We extended three SPARQL endpoint federation engines with HiBISCuS: DARQ [10] (index-only), FedX [14] (index-free), and SPLENDID [4] (hybrid). In each of the extensions, we only replaced the source selection with HiBISCuS. The query execution mechanisms remained unchanged. We compared our best extension (i.e., SPLENDID+HiBISCuS) with ANAPSID as this engine showed competitive results w.r.t. its index compression and number of TPW sources selected.

Metrics: We compared the three engines against their HiBISCuS extension. For each query we measured (1) the total number of TPW sources selected, (2) the total number of SPARQL ASK requests submitted during the source selection, (3) the average source selection time and (4) the average query execution time. We also compare the source index/data summaries generation time and index compression ratio of various state-of-the art source selection approaches.

\subsection{Experimental Results}

Index Construction Time and Compression Ratio Table 2 shows a comparison of the index/data summaries construction time and the compression ratio ${ }^{9}$ of various stateof-the art approaches. A high compression ratio is essential for fast index lookup during source selection. HiBISCuS has an index size of $458 \mathrm{~KB}$ for the complete FedBench data dump (19.7 GB), leading to a high compression ratio of $99.99 \%$. The other approaches achieve similar compression ratios. HiBISCuS's index construction time is second only to ANAPSID's. This is due to ANAPSID storing only the distinct predicates in its index. Our results yet suggest that our index containing more information is beneficial to the query execution time on FedBench.

\footnotetext{
${ }^{9}$ The compression ratio is given by (1 - index size/total data dump size).
} 
Table 2: Comparison of index construction time and compression ratio. QTree's compression ratio is taken from [5]. (NA = Not Applicable).

\begin{tabular}{l|c|c|c|c|c|c|c}
\hline & FedX & SPLENDID & LHD & DARQ & ANAPSID & Qtree & HiBISCuS \\
\hline Index Generation Time (min) & NA & 75 & 75 & 102 & 6 & - & 36 \\
Compression Ratio (\%) & NA & 99.998 & 99.998 & 99.997 & 99.999 & 96 & 99.997 \\
\hline
\end{tabular}

Efficient Source Selection We define efficient source selection in terms of three metrics: (1) the total number of TPW sources selected, (2) total number of SPARQL ASK requests used to obtain (1), and (3) the TPW source selection time. Table 3 shows a comparison of the source selection approaches of FedX, SPLENDID, ANAPSID and HiBISCuS based on these three metrics. Note that FedX (100\% cached) means that we gave FedX enough memory to use only its cache to perform the complete source selection. This is the best-case scenario for FedX. Overall, HiBISCuS (ASK-dominant) is the most efficient approach in terms of total TPW sources selected, HiBISCuS (Index-dominant) is the most efficient hybrid approach in terms of total number of ASK request used, and FedX (100\% cached) is most efficient in terms of source selection time. However, FedX (100\% cached) clearly overestimates the set of sources that actually contributes to the final result set of query. In the next section, we will see that this overestimation of sources greatly leads to a slightly higher overall query runtime. For ANAPSID, the results are based on Star-Shaped Group Multiple endpoint selection (SSGM) heuristics presented in its extension [9]. Further, the source selection time represents the query decomposition time as both of these steps are intermingled.

Query execution time The most important criterion when optimizing federated query execution engines is the query execution time. Figures 4, 5, and 6 show the results of our query execution time experiments. Our main results can be summarized as follows:

(1) Overall, the ASK-dominant (AD) version of our approach performs best. $\mathrm{AD}$ is on average (over all 25 queries and 3 extensions) $27.82 \%$ faster than the index-dominant (ID) version. The reason for this improvement is due to ID overestimating sources in some queries. For example, in CD1, AD selects the optimal number of sources (i.e., 4) while ID selects 12 sources. In some cases, the overestimation of sources by ID also slows down the source pruning (e.g. CD2),

(2) A comparison of our extensions with $\mathrm{AD}$ shows that all extensions are more time-efficient than the original systems. In particular, FedX's (100\% cached) runtime is improved in 20/25 queries (net query runtime improvement of 24.61\%), FedX's (cold) is improved in 25/25 queries (net improvement: 53.05\%), SPLENDID 's is improved in 25/25 queries (net improvement: $82.72 \%$ ) and DARQ's is improved in 21/21 (2 queries are not supported, 1 query time out, and 1 query runtime error) queries (net improvement: $92.22 \%$ ). Note that these values were computed only on those queries that did not timeout. Thus, the net improvement brought about by AD is actually even better than the reported values. The reason for our slight (less than $5 \mathrm{msec}$ ) greater runtime for $5 / 25$ queries in FedX (100\% cached) is due to FedX (100\% cached) already selecting the optimal sources for these queries. Thus, the overhead due to our pruning of the already optimal list of sources affects the overall query runtime. 
Table 3: Comparison of the source selection in terms of total TPW sources selected \#T, total number of SPARQL ASK requests \#A, and source selection time $\mathbf{S T}$ in msec. ST* represents the source selection time for FedX(100\% cached i.e. $\# \mathrm{~A}=0$ for all queries) which is very rare in practical. ST** represents the source selection time for HiBISCuS $(\mathrm{AD}$, warm) with \#A =0 for all queries. $(\mathbf{A D}=\mathrm{ASK}$-dominant, $\mathbf{I D}=$ index-dominant, $\mathbf{Z R}=$ Zero results, $\mathbf{N S}=$ Not supported, $\mathbf{T} / \mathbf{A}=$ Total/Avg., where Total is for \#T, \#A, and Avg. is ST, $\mathrm{ST}^{*}$, and $\mathrm{ST}^{* *}$ )

\begin{tabular}{|c|c|c|c|c|c|c|c|c|c|c|c|c|c|c|c|c|c|c|c|c|}
\hline & \multicolumn{4}{|c|}{ FedX } & \multicolumn{3}{|c|}{ SPLENDID } & \multicolumn{3}{|c|}{ DARQ } & \multicolumn{3}{|c|}{ ANAPSID } & \multicolumn{4}{|c|}{ HiBISCuS(AD) } & \multicolumn{3}{|c|}{ HiBISCuS(ID) } \\
\hline Qry & \#T & \#A & ST & ST* & \#T & $\# \mathrm{~A}$ & ST & \#T & \#A & ST & \#T & \#A & ST & \#T & \#A & ST & ST** & \#T & \#A & ST \\
\hline$\overline{\mathrm{CD} 1}$ & 11 & 27 & 285 & 6 & 11 & 26 & 392 & $\mathrm{NS}$ & NS & NS & 3 & 20 & 667 & 4 & 18 & 215 & 36 & 12 & \begin{tabular}{|l|}
$\mathbf{0}$ \\
\end{tabular} & 363 \\
\hline $\mathrm{CD} 2$ & 3 & 27 & 200 & 6 & 3 & 9 & 294 & 10 & $\mathbf{0}$ & 6 & 3 & 1 & 42 & 3 & 9 & 4 & 3 & 3 & $\mathbf{0}$ & 57 \\
\hline CD3 & 12 & 45 & 367 & 8 & 12 & 2 & 304 & 20 & $\mathbf{0}$ & 12 & 5 & 2 & 73 & 5 & $\mathbf{0}$ & 77 & 41 & 5 & $\mathbf{0}$ & 91 \\
\hline CD4 & 19 & 45 & 359 & 8 & 19 & 2 & 310 & 20 & $\mathbf{0}$ & 12 & 5 & 3 & 128 & 5 & $\mathbf{0}$ & 54 & 52 & 5 & $\mathbf{0}$ & 179 \\
\hline CD5 & 11 & 36 & 374 & 7 & 11 & 1 & 313 & 11 & $\mathbf{0}$ & 4 & 4 & 1 & 66 & 4 & $\mathbf{0}$ & 25 & 23 & 4 & $\mathbf{0}$ & 58 \\
\hline CD6 & 9 & 36 & 316 & 8 & 9 & 2 & 298 & 10 & $\mathbf{0}$ & 11 & 10 & 11 & 140 & 8 & $\mathbf{0}$ & 36 & 23 & 8 & $\mathbf{0}$ & 54 \\
\hline CD7 & 13 & 36 & 324 & 9 & 13 & 2 & 335 & 13 & 0 & 6 & 6 & 5 & ZR & 6 & 0 & 30 & 35 & 6 & 0 & 55 \\
\hline LS1 & $\mathbf{1}$ & 18 & 248 & 9 & $\mathbf{1}$ & $\mathbf{0}$ & 217 & $\mathbf{1}$ & 0 & 4 & 1 & 0 & 35 & 1 & 0 & 5 & 6 & 1 & 0 & 9 \\
\hline LS2 & 11 & 27 & 264 & 8 & 11 & 26 & 390 & NS & NS & NS & 12 & 30 & 548 & 7 & 18 & 118 & 60 & 7 & 0 & 118 \\
\hline LS3 & 12 & 45 & 413 & 8 & 12 & 1 & 310 & 20 & 0 & 9 & 5 & 13 & 808 & 5 & 0 & 31 & 27 & 5 & 0 & 200 \\
\hline LS4 & 7 & 63 & 445 & 7 & 7 & 2 & 287 & 15 & 0 & 15 & 7 & 1 & 314 & 7 & 0 & 8 & 9 & 7 & 0 & 15 \\
\hline LS5 & 10 & 54 & 440 & 8 & 10 & 1 & 308 & 18 & 0 & 13 & 7 & 4 & 885 & 8 & 0 & 20 & 21 & 8 & 0 & 44 \\
\hline LS6 & 9 & 45 & 430 & 8 & 9 & 2 & 347 & 17 & 0 & 7 & 5 & 13 & 559 & 7 & 0 & 23 & 22 & 7 & 0 & 42 \\
\hline LS7 & 6 & 45 & 389 & 8 & 6 & 1 & 292 & 6 & 0 & 5 & 7 & 2 & 193 & 6 & 0 & 18 & 17 & 6 & 0 & 24 \\
\hline$\overline{\mathrm{LD} 1}$ & 8 & 27 & 297 & 8 & 8 & 1 & 295 & 11 & 0 & 7 & 3 & 1 & 428 & 3 & $\mathbf{0}$ & 24 & 19 & 3 & 0 & 21 \\
\hline LD2 & 3 & 27 & 320 & 7 & 3 & 1 & 268 & 3 & 0 & 9 & 3 & 0 & 34 & 3 & 0 & 3 & 5 & 3 & 0 & 6 \\
\hline LD3 & 16 & 36 & 330 & 9 & 16 & 1 & 324 & 16 & 0 & 11 & 4 & 2 & 130 & 4 & 0 & 31 & 29 & 4 & 0 & 48 \\
\hline LD4 & 5 & 45 & 326 & 7 & 5 & 2 & 290 & 5 & 0 & 17 & 5 & 0 & 33 & 5 & 0 & 6 & 7 & 5 & 0 & 10 \\
\hline LD5 & 5 & 27 & 280 & 8 & 5 & 2 & 236 & 13 & 0 & 4 & 3 & 2 & 210 & 3 & 0 & 9 & 9 & 3 & 0 & 19 \\
\hline LD6 & 14 & 45 & 385 & 8 & 14 & 1 & 331 & 14 & 0 & 8 & 14 & 12 & 589 & 7 & 0 & 32 & 30 & 7 & 0 & 136 \\
\hline LD7 & 3 & 18 & 258 & 7 & 3 & 2 & 235 & 4 & 0 & 4 & 2 & 4 & 223 & 4 & 0 & 7 & 7 & 4 & 0 & 11 \\
\hline LD8 & 15 & 45 & 337 & 8 & 15 & 1 & 333 & 15 & 0 & 7 & 9 & 7 & 1226 & 5 & 0 & 23 & 25 & 5 & 0 & 41 \\
\hline LD9 & 3 & 27 & 228 & 12 & 3 & 5 & 188 & 6 & 0 & 3 & 3 & 3 & 1052 & 3 & 9 & 50 & 3 & 3 & 0 & 17 \\
\hline LD10 & 10 & 27 & 274 & 8 & 10 & 2 & 309 & 11 & 0 & 6 & 3 & 4 & 2010 & 3 & 0 & 19 & 18 & 3 & 0 & 27 \\
\hline LD11 & 15 & 45 & 351 & 7 & 15 & 1 & 260 & 15 & 0 & 9 & 5 & 2 & 2904 & 7 & 0 & 23 & 24 & 7 & 0 & 42 \\
\hline T/A & 231 & 918 & 330 & 8 & 231 & 96 & 299 & 274 & O & 8 & 134 & 143 & 554 & 123 & 54 & 36 & 22 & 131 & O & 67 \\
\hline
\end{tabular}

(3) Our extensions allow some queries that timed out to be carried out before the time-out. This is especially the case for our DARQ extension, where LD6 and LD10 are carried out in $1123 \mathrm{msec}$ and $377 \mathrm{msec}$ respectively by DARD+AD, while they did not terminate within the time-out limit of 30 minutes on the original system.

(4) Our SPLENDID (AD) extension is $98.91 \%$ faster than ANAPSID on 24 of the 25 queries. For CD7, ANAPSID returned zero results.

An interesting observation is that FedX(100\%) is better than SPLENDID in 25/25 queries and $58.17 \%$ faster on average query runtime. However, our AD extension of SPLENDID is better than AD extension of FedX(100\%) in 20/25 queries and $45.20 \%$ faster on average query runtime. This means that SPLENDID is better than FedX in term of pure query execution time (excluding source selection time). A deeper investigation of the runtimes of both systems shows that SPLENDID spends on average 56.10\% of total query execution on source selection. Thus, our extension showcase clearly that an 


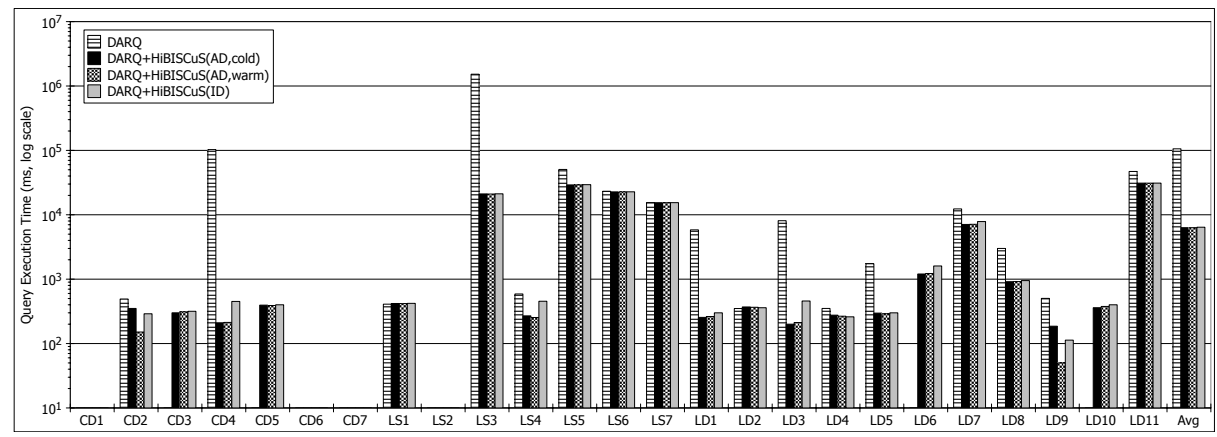

Fig. 4: Query runtime of DARQ and its HiBISCuS extensions. CD1, LS2 not supported, CD6 runtime error, $\mathrm{CD} 7$ time out for both. LD6, LD10 timeout and CD3 runtime error for DARQ.

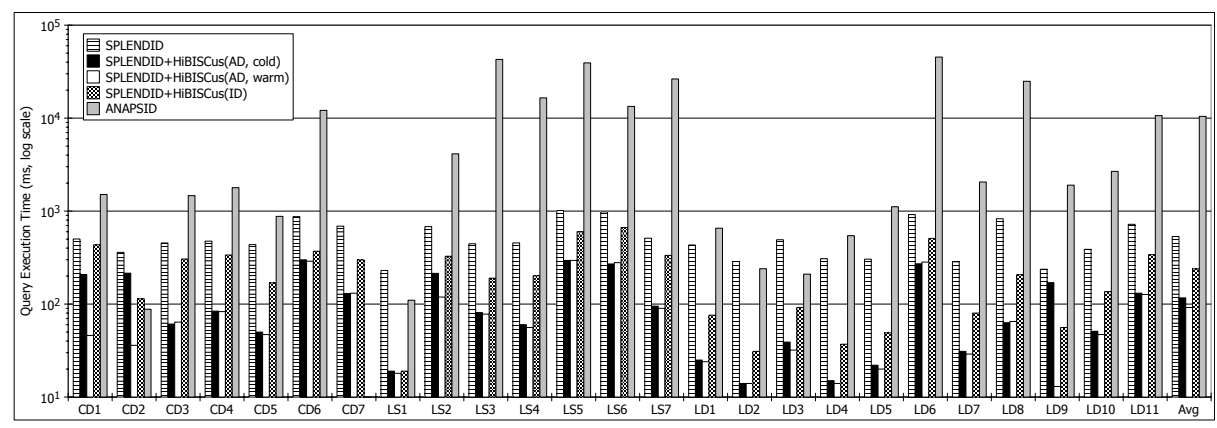

Fig. 5: Query runtime of ANAPSID, SPLENDID and its HiBISCuS extensions. We have zero results for ANAPSID CD7

efficient source selection is one of key factors in the overall optimization of federated SPARQL query processing.

\section{Conclusion and Future Work}

In this paper we presented HiBISCus, a labelled hypergraph based approach for efficient source selection for SPARQL endpoint federation. We evaluated our approach against DARQ, SPLENDID, FedX and ANAPSID. The evaluation shows that the query runtime of the first three systems is improved significantly on average.

In future, we will investigate the impact of the threshold $\theta$ on our approach. We will also study the effect of our source pruning algorithm on SPARQL 1.1 queries with SPARQL service clause, where the TPW sources are already specified by the user. Furthermore, we will evaluate our approach on big data as the query execution time for majority of the FedBench queries is less than 1s, which make it difficult to select the best SPARQL federation engine and have have a deeper look into the behaviour of these engines in different data environments. 


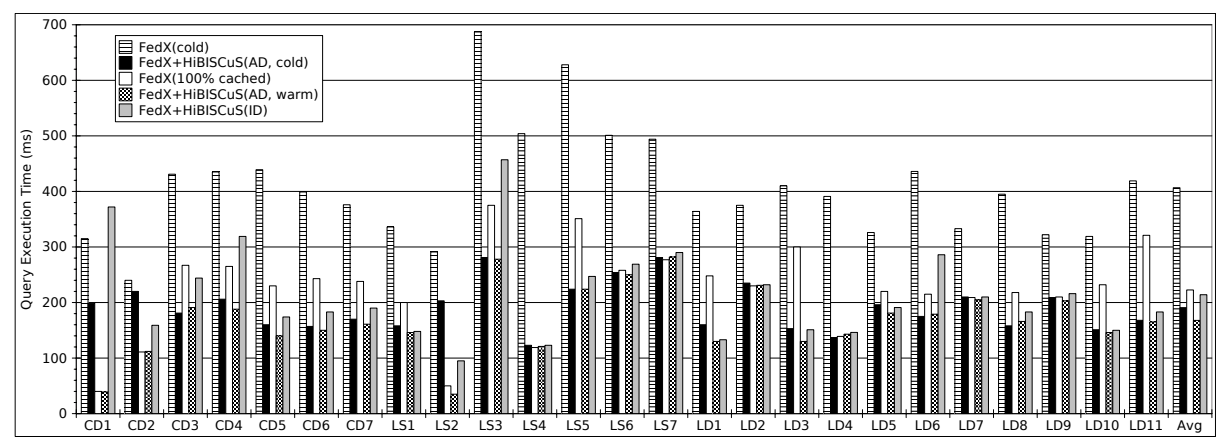

Fig. 6: Query runtime of FedX and its HiBISCuS extensions

\section{Acknowledgments}

This work was partially financed by the FP7 project GeoKnow (GA no. 318159).

\section{References}

1. M. Acosta, M.-E. Vidal, T. Lampo, J. Castillo, and E. Ruckhaus. ANAPSID: an adaptive query processing engine for SPARQL endpoints. In ISWC, 2011.

2. Z. Akar, T. G. Halaç, E. E. Ekinci, and O. Dikenelli. Querying the web of interlinked datasets using void descriptions. In LDOW at WWW, 2012.

3. S. Auer, J. Lehmann, and A.-C. Ngonga Ngomo. Introduction to linked data and its lifecycle on the web. In Reasoning Web, 2011.

4. O. Görlitz and S. Staab. Splendid: Sparql endpoint federation exploiting void descriptions. In COLD at ISWC, 2011.

5. A. Harth, K. Hose, M. Karnstedt, A. Polleres, K.-U. Sattler, and J. Umbrich. Data summaries for on-demand queries over linked data. In $W W W, 2010$.

6. Z. Kaoudi, M. Koubarakis, and K. Kyzirakos. Atlas: Storing, updating and querying rdf(s) data on top of dhts. JWS, 8(4), 2010.

7. G. Ladwig and T. Tran. Linked data query processing strategies. In ISWC. 2010.

8. S. Lynden, I. Kojima, A. Matono, and Y. Tanimura. Aderis: An adaptive query processor for joining federated sparql endpoints. In OTM. 2011.

9. G. Montoya, M.-E. Vidal, and M. Acosta. A heuristic-based approach for planning federated sparql queries. In COLD, 2012.

10. B. Quilitz and U. Leser. Querying distributed rdf data sources with sparql. In ESWC, 2008.

11. M. Saleem, A.-C. Ngonga Ngomo, J. X. Parreira, H. F. Deus, and M. Hauswirth. Daw: Duplicate-aware federated query processing over the web of data. In ISWC, 2013.

12. M. Saleem, S. Shanmukha, A.-C. Ngonga, J. S. Almeida, S. Decker, and H. F. Deus. Linked cancer genome atlas database. In I-Semantics 2013, 2013.

13. M. Schmidt, O. Görlitz, P. Haase, G. Ladwig, A. Schwarte, and T. Tran. Fedbench: a benchmark suite for federated semantic data query processing. In ISWC, 2011.

14. A. Schwarte, P. Haase, K. Hose, R. Schenkel, and M. Schmidt. Fedx: Optimization techniques for federated query processing on linked data. In $I S W C, 2011$.

15. X. Wang, T. Tiropanis, and H. C. Davis. Lhd: Optimising linked data query processing using parallelisation. In $L D O W$ at $W W W, 2013$. 